SPECTRAL EVOLUTION OF HM SAGITTAE IN THE ULTRAVIOLET

Ch. Kindl and H. Nussbaumer

Institute of Astronomy, ETH-Zentrum, CH-8092 Zl̈rich, Switzerland

We have observed the ultraviolet spectrum of HM Sge with the International Ultraviolet Explorer during 1978-1980. The low resolution spectra are shown in Figure 1.

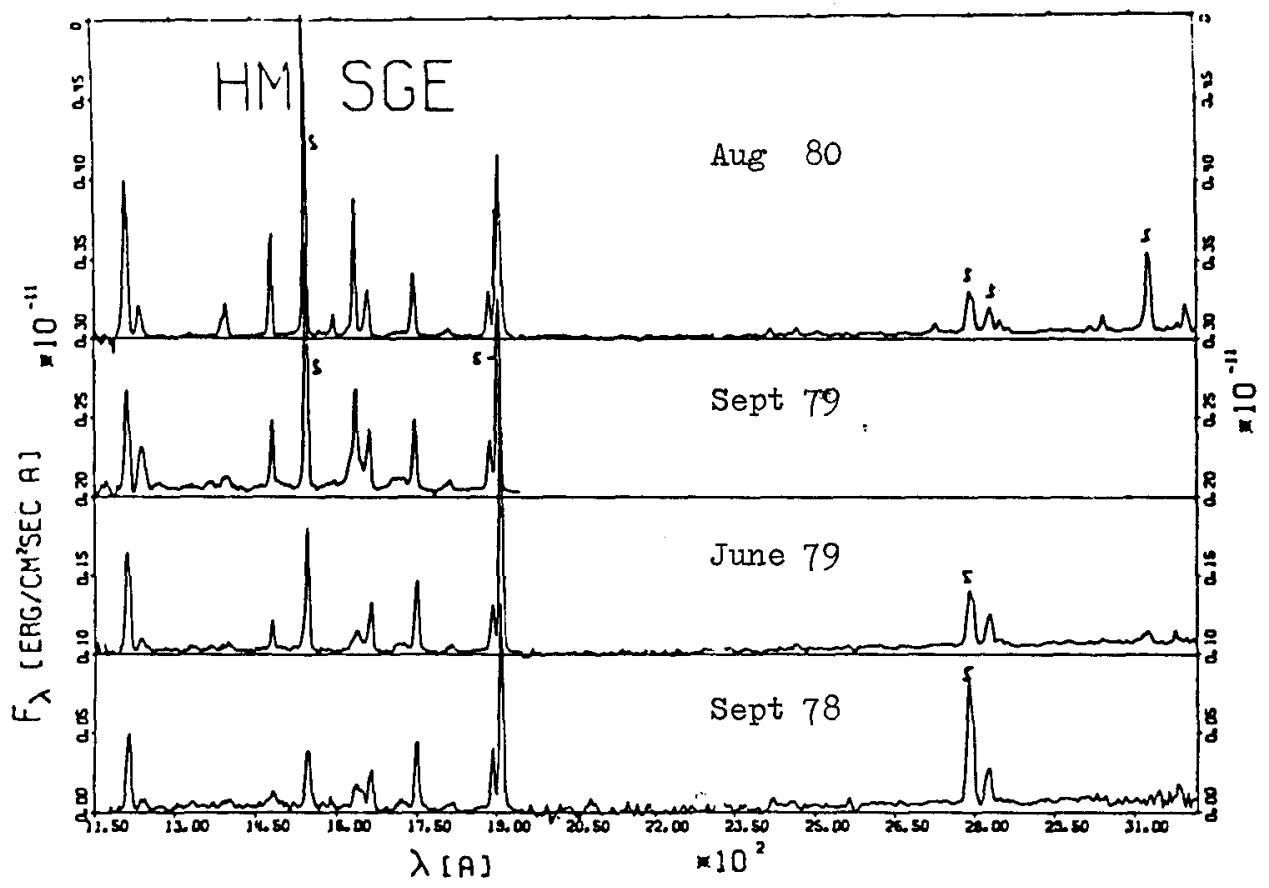

Figure 1. The low resolution ultraviolet spectrum of HM Sge during 19781980.

$M$. Friedjung and $R$. Viotti (eds.), The Nature of Symbiotic Stars, 213-214. Copyright $\odot 1982$ by D. Reidel Publishing Company. 
There are strang changes over the two years. The fluxes of OIII] $\lambda 1664, \operatorname{NIII}] \lambda 1750, \operatorname{SiIII}] \lambda 1892, \mathrm{CIII}] \lambda 1908$, and the CII/OIII blend at $\lambda 2323$ have remained approximately constant, but the blend of OIV] and SiIV at $\lambda 1400, N I V] \lambda 1485, \operatorname{CIV} \lambda 1549$, and HeII $\lambda 1640$ increased their fluxes by approximately a factor 3 , so did the Bowen line at $\lambda 3133$. NV $\lambda 1240$ decreased after an initial increase. The change was particular1y rapid between June and september 1979 .

The interpretation of these spectra has only started, it will rest heavily on high resolution spectra which we took as well. We intend to continue these observations; we hope that well calibrated spectra in other wavelength domains will be taken as well.

\section{DISCUSSION ON HM SAGITTAE}

Ciatti: Since the many similarities between HM Sge and V1016 Cyg have been often remarked upon, it is also to be recalled that:

V1016 Cyg showed a very smooth photometric and spectroscopic evolution; HM Sge has presented several phases of higher and lower excitation (in the general trend leading to higher ionization stages; now HeII and some times [FeVII]), and light variations around "maximum" light.

Recent variations in the optical spectrum of HM Sge are reported by Ciatti et al. (1979, Astr. Astrophys. 79, 247) and Andrillat, Ciatti and Swings, Astr. Astrophys. submitted. Furthermore, [FeVII] is not recorded during July-August 1981 on the Asiago plates.

Keyes: Dr. Kwok mentioned that Wolf-Rayet characteristics existed in HM Sge. Are these well-defined, do they indicate a WN or a WC type? (This has a potentially important bearing on the evolutionry state of the system from the standpoint of what types of nuclear processing may have occurred).

Kwok: HM Sge has been classified as WN6 by Ciatti et al. (1978, Astr. Astrophys. 68, 751), whereas Brown et al. (1978, Ap. Letters 19, 75) call it WC. 